\title{
Réforme des structures intermédiaires de santé en République démocratique du Congo
}

\author{
Reorganization of the provincial health system in the Democratic \\ Republic of the Congo
}

Jean Bosco Kahindo Mbeva ${ }^{1}$, Carole Schirvel ${ }^{2}$, Eliane Godelet ${ }^{3}$, Alain Wodon ${ }^{1}$, Denis Porignon ${ }^{4}$, Michel Bonami ${ }^{5}$

\section{$[\rightarrow$ Résumé}

Introduction : Dans le cadre de la réforme du système de santé et de la décentralisation prévue par la constitution en République démocratique du Congo (RDC), cette étude présente le processus et les résultats obtenus en termes de modèle des structures intermédiaires de santé.

Méthodes : Recherche-action participative multidisciplinaire en deux phases et neuf étapes - intégrant des méthodes d'analyse organisationnelle, de team building et d'apprentissage organisationnel - qui a permis de tester en temps réel des actions de transformation, et de capitaliser les savoirs ainsi générés sous la forme d'un modèle innovant d'organisation.

Résultats : Cette recherche-action a mis en évidence des dispositifs et des modes d'action rendant possible une action collective orientée sur le support du niveau intermédiaire aux districts de santé et qui permet de pallier les faiblesses de l'organigramme officiel. La transformation organisationnelle a ensuite été formalisée dans un modèle innovant d'organisation basé sur quatre métiers : (i) Appui aux districts de santé, (ii) Information sanitaire, recherche et communication, (iii) Inspection-contrôle, (iv) Gestion des ressources. Les quatre métiers et leurs services ont été définis, les compétences et activités liées décrites. Les résultats, en termes de métiers, ont été intégrés au nouveau plan national de développement sanitaire.

Conclusions : La démarche adoptée, centrée sur l'apprentissage et le changement organisationnels, a elle-même contribué à redynamiser certaines fonctions du Niveau intermédiaire. Deux défis sont encore à relever : accompagner la transformation et l'étendre aux autres provinces de la RDC selon une approche participative, condition nécessaire d'ajustement aux contextes spécifiques.

Mots-clés: Modèles d'organisation; Politique de santé; Recherche empirique; Recherche sur les services de santé; Réforme des soins de santé; République démocratique du Congo.

\section{$\hookrightarrow$ Summary}

Introduction: In the framework of implementation of health system reform in the Democratic Republic of the Congo (DRC), and in a context of decentralization dictated by the National Constitution, this study presents the process and results obtained in terms of the provincial level of health care organization in DRC. Methods: A two-year multidisciplinary interventional research protocol was elaborated with two phases and 9 steps including organizational analysis, team building, and organizational learning. It resulted in transformational actions and improved knowledge, allowing the development of an innovative organizational model of the intermediate level of the health care system in the Democratic Republic of the Congo.

Results: This interventional research gave rise to function plans set up by the provincial health division (PHD) in order to develop a more participative management and to compensate for the weaknesses of the current structural organization. Experts provided support to PHD for implementation of a new structure in order to institutionalize this new participative management. The new organizational structure of the PHD is based on 4 professions: i) health district support; ii) control and inspection; iii) information, communication and research and iv) resources management. PHD and experts defined these professions and described the required skills. Results were integrated into the new national health plan.

Conclusions: Apart from the concrete results obtained, two major challenges need to be addressed: i) support the transformation of PHD from the current situation to the new model and ii) extend this new model to the other provinces, according to the same participative approach, a necessary condition to adjust the organization flow-chart to the context.

Keywords: Models, organizational; Health policy; Empirical research; Healthcare reform; Health services research; Democratic Republic of the Congo.

\footnotetext{
${ }^{1}$ Centre scientifique et médical - Université Libre de Bruxelles (CEMUBAC) - Route de Lennik, 808 - CP 595 - B-1070 Bruxelles - Belgique.

${ }^{2}$ Fédération Wallonie Bruxelles - Direction de la surveillance et de la protection de la santé - Cellule de surveillance des maladies infectieuses

- Belgique.

${ }^{3}$ FOPES (UCL) - Belgique.

${ }^{4}$ Université de Liège (ULG) - Belgique.

${ }^{5}$ Université Catholique de Louvain (UCL) - Belgique.
} 


\section{Introduction}

Le renouveau des soins de santé primaires lancé en 2008 par l'OMS impose des réformes profondes des systèmes de santé des pays tant du Nord que du Sud [1]. Ces réformes visent une meilleure congruence entre l'organisation des systèmes de santé et les objectifs poursuivis, une des six fonctions essentielles du pilotage des systèmes de santé $[2,3]$. Cette congruence organisationnelle est un préalable à l'efficacité et à l'efficience des systèmes de santé. Pour de nombreux pays africains, les modes d'organisation des systèmes de santé sont généralement bureaucratiques $[4,5]$ et peu compatibles avec la complexité des systèmes de santé contemporains [4-6].

La République démocratique du Congo (RDC) ne fait pas exception. Son système de santé, bien que basé sur l'approche des soins de santé primaires, renvoie à une bureaucratie. Jusqu'en 2008, il comptait, rien qu'au niveau central, 65 directions, dont 52 en charge des programmes spécialisés. Ces directions étaient répliquées sous forme de bureaux et de coordinations des programmes spécialisés au niveau provincial ou «niveau intermédiaire du système sanitaire » (NISS). Les missions assignées au NISS se résumaient, jusqu'en 2008, au support du niveau opérationnel constitué de 515 zones de santé ou districts de santé selon l'OMS. Dans la suite de cet article, nous proposons de parler du district de santé à la place de la zone de santé. Comme l'indiquent le classement par l'OMS [7] et une évaluation menée en RDC au cours de la décennie 2000 [8], le système de santé de la RDC s'est montré inadapté. Entre la fin des années 90 et le début des années 2000, il a été sévèrement impacté par les crises socioéconomiques et humanitaires récurrentes et a montré son incapacité à répondre aux besoins de santé des populations congolaises. En 2006, la RDC s'est engagée à réformer son système de santé ; c'est ainsi que la stratégie de réforme du système de santé (SRSS), élaborée par des cadres congolais, dont ceux du Ministère de la Santé, a été rendue publique fin 2006. Un des axes de cette stratégie prévoit explicitement de réformer le NISS. La finalité de cette réorganisation est de soutenir la revitalisation du district de santé, priorité conditionnelle au renforcement du système de santé congolais $[8,9]$. Pour avancer dans le sens de la réforme du NISS, au moins deux défis devaient être relevés: i) que le nouveau mode d'organisation prenne en compte la complexité des systèmes de santé en termes de particularités épidémiologiques, de prestations des services, de gestion des ressources humaines et financières et d'information sanitaire dans un environnement pour le moins difficile [4-6] ; ii) que le nouveau mode d'organisation soit accepté par les principaux acteurs pour être mis en œuvre.

Certains auteurs ont montré qu'il était possible de mobiliser les acteurs de santé dans des processus d'innovation et de construction d'outils pertinents pour leur action [10]. Ces processus se réfèrent, en partie, à l'apprentissage productif « en double ou triple boucle», décrit par Takeuchi et Nonaka [11]. D'après nos recherches bibliographiques ayant utilisé comme mots-clés " health system reform » or "health system design» and "Africa» sur Pudmed, peu d'études ont documenté les processus d'élaboration des modes d'organisations du Niveau intermédiaire des systèmes de santé dans les contextes africains. Nous formulons l'hypothèse que l'apprentissage productif peut être mis en œuvre dans le cadre d'une recherche-action pour concevoir des structures organisationnelles cohérentes en contextes africains.

L'objectif de cet article est de présenter le processus de construction et les résultats obtenus en tant que modèle innovant d'organisation des structures intermédiaires du secteur de la santé en RDC.

\section{Méthodes}

Cette étude concerne les structures intermédiaires de santé en RDC. La méthodologie de l'étude est basée sur (i) un terrain de recherche-action, (ii) une équipe multidisciplinaire et (iii) des méthodes de recherche-action participative et de team-building.

\section{Terrain de recherche-action}

L'étude a été menée de 2008 à 2010 en RDC au niveau de deux provinces couvrant près de six millions d'habitants chacune : le Nord Kivu et le Kasaï Oriental. Le choix de ces deux provinces a été dicté par leurs caractéristiques du fonctionnement du système de santé : le Nord Kivu considéré comme l'une des provinces plus avancées dans l'organisation des services de santé, le Kasaï Oriental plus représentatif du niveau global d'avancement des autres provinces de la RDC. La recherche a été menée alors que la RDC s'engageait dans un processus de forte décentralisation vers le niveau provincial prônée par sa nouvelle constitution promulguée également en 2006. 


\section{L'équipe multidisciplinaire de recherche}

L'équipe de recherche était multidisciplinaire et constituée de 10 personnes, des acteurs de terrain et des chercheurs : une anthropologue $(\mathrm{N}=1)$, des médecins de santé publique spécialisés dans le renforcement des systèmes de santé $(\mathrm{N}=3)$ et expérimentés en gestion des systèmes de santé ( $\mathrm{N}=2$ ). L'équipe s'est également adjoint l'expertise de spécialistes en systèmes d'informations sanitaires $(\mathrm{N}=1)$ et en formation d'adultes $(\mathrm{N}=1)$ et d'un juriste $(\mathrm{N}=1)$. Cette équipe a été guidée par un chercheur psychosociologue spécialiste des questions d'intervention au niveau des organisations $(\mathrm{N}=1)$.

\section{Une recherche-action selon une approche participative et de team-building en deux phases et neuf étapes (figure 1)}

La première phase : elle est adaptée du modèle de recherche sur les systèmes de santé [12] et intègre les principes d'apprentissage organisationnel [13]. La recherche est partie d'un modèle simple de structure provinciale de santé qui soutient les districts de santé dans leur développement. Sur cette base, un état des lieux des structures du Niveau intermédiaire du système de santé (NISS) - encore appelées structures intermédiaires dans le texte - a été menée en 2008 au Nord Kivu et au Kasaï Oriental (Étape 1).

Les données ont été collectées par des entretiens individuels semi-directifs auprès des cadres du NISS, des cadres des districts sanitaires et des partenaires. Cette collecte des données a été suivie d'une analyse participative. Cet état des lieux a été mené par un psychosociologue des organisations et une socio-anthropologue, en collaboration avec des médecins de santé publique. Sur la base des résultats d'analyses, l'équipe de recherche a mis en évidence des modes d'action habituels, notamment ceux qui ont rendu une action collective possible au niveau de deux NISS. En outre, les actions d'amélioration et les résultats escomptés adaptés aux contextes et aux attentes des acteurs concernés

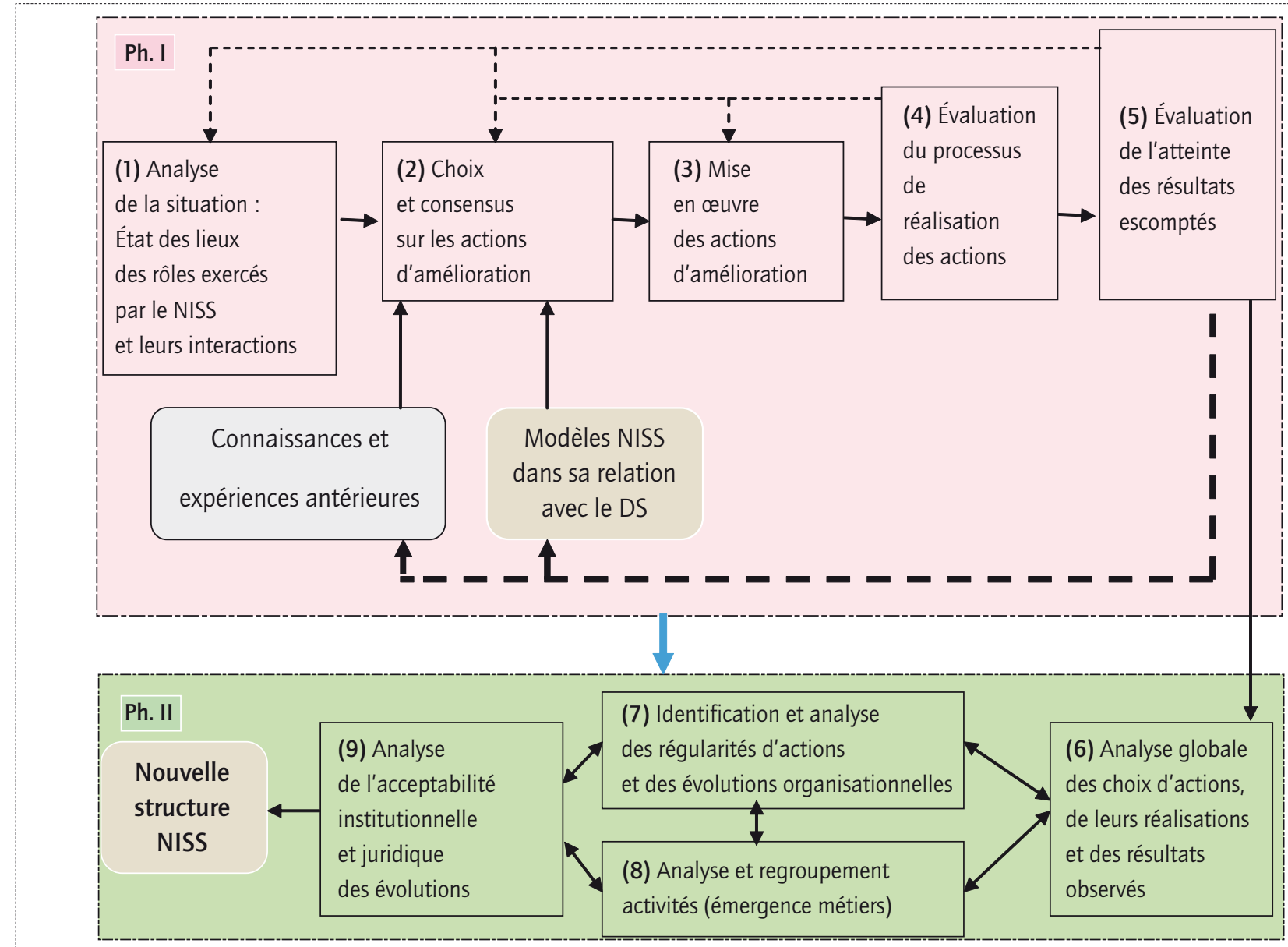

Figure 1 : Schéma du processus global de Recherche-Action 
pour chacune des deux provinces ont été identifiées (Étape 2). Sur cette base, des actions d'amélioration notamment de la coordination interne et externe, de l'accompagnement des équipes des districts de santé, de la gestion des financements, ont été menées (étapes 3). Avec les acteurs de terrain dans les deux provinces, les médecins de santé publique ont identifié les indicateurs de suivi de la mise en œuvre des actions envisagées et d'évaluation des résultats escomptés. Les instances de collecte de données ont été identifiées et les outils de collecte élaborés par les mêmes acteurs. Les évaluations trimestrielles des activités (Étape 4) et des résultats (Étape 5) ont été menées conjointement par les équipes de terrain et les membres de l'équipe de recherche présents en provinces. Ces évaluations ont intégré une approche réflexive et ont permis soit d'ajuster les processus de mise en œuvre des actions, soit de décider de nouvelles actions à mener. Elles ont également permis de mieux comprendre les facteurs qui influencent le résultat de telle action ou de tel dispositif. Ces évaluations ont enfin permis d'identifier les actions et les modes d'organisation qui rendent possible une réelle implication des acteurs du NISS dans le soutien des districts de santé de la Province. Ces enseignements ont permis d'affiner le modèle de départ. Ce processus a été itératif entre 2008 et 2010 et capitalisé sur le terrain par les médecins de santé publique, en lien avec les autres membres de l'équipe de recherche non présents de manière permanente dans les deux provinces. Notons que le psychosociologue, le socio-anthropologue, le spécialiste en système d'information sanitaire, le spécialiste en formation d'adultes et le juriste ne résidaient pas de manière permanente au niveau des provinces.

La deuxième phase concerne le processus de formalisation des transformations du NISS sous forme de modèle d'organisation qui impulse le développement du district de santé. Cette phase a été conduite conjointement par la spécialiste en formation d'adultes et le psychosociologue des organisations, avec la participation active des médecins de santé publique et des acteurs de terrain en province. Elle a débuté par une analyse et une consolidation des enseignements ayant émergé lors de la première phase (Étape 6). Les évolutions des modes d'organisation et des dispositifs opérationnels ont été repérées et analysées (Étape 7). Les activités qui leur sont liées, ont été cernées et regroupées, faisant ainsi émerger des «métiers » (Étape 8). Le métier est compris ici comme : "un ensemble évolutif de situations professionnelles auquel est associé un corpus de valeurs et de ressources pour pouvoir le gérer» [14]. Cette étape s'est inspirée des approches de l'analyse de l'activité $[11,15]$ et d'apprentissage organisationnel [14].
Chaque métier (4) a été explicité en termes de compétences, des services et unités, et d'effectifs nécessaires. Cette deuxième phase a été clôturée par une validation institutionnelle et juridique de la transformation organisationnelle ainsi produite (Étape 9). Un expert juriste a été mobilisé pour analyser les transformations ayant émergé du processus. Des « aller-retour » ont eu lieu entre l'équipe de recherche et les instances nationales et provinciales du ministère de la Santé, les comités provinciaux de pilotage sanitaire et d'autres parties prenantes à la réorganisation des structures intermédiaires de santé en RDC. Ces transformations ont été formalisées sous forme de fascicules afin de clarifier le modèle proposé.

\section{Résultats}

Cette recherche-action a permis une mise en œuvre effective d'actions d'amélioration au niveau des structures intermédiaires de deux provinces. Elle a fait émerger les dispositifs opérationnels en leur sein et formalisé de nouvelles modalités d'organisation susceptibles de pallier les faiblesses d'une ancienne organisation mécaniste qui est à la fois peu flexible, verticale et inefficace.

\section{Les actions d'amélioration et structurantes du NISS au Nord Kivu (NK) et au Kasaï Oriental (Kor)}

Les actions d'amélioration ont été menées dans quelques domaines dont : la gestion des financements, la supervision du personnel de santé de district, la coordination en interne des structures intermédiaires et la coordination externe avec les partenaires. Le tableau I illustre quelques actions d'amélioration, les effets attendus, ceux observés et les ajustements réalisés.

\section{Les dispositifs opérationnels}

Ces dispositifs comprennent des instances internes de travail entre cadres du NISS (équipe cadre provinciale, groupes de travail, cellule de gestion), et des instances de travail avec les autres acteurs du système sanitaire au niveau provincial (commissions, comité de pilotage du financement, comité provincial de pilotage sanitaire, les revues des activités des soins de santé primaires). La Centrale régionale de distribution des médicaments, le bulletin d'informations 
Tableau I : Quelques actions d'amélioration entreprises au niveau des NISS Nord Kivu et Kasaï Oriental

\begin{tabular}{|c|c|c|c|c|}
\hline Actions décidées & Effets escomptés & Actions réalisées & Effets observés & $\begin{array}{l}\text { Ajustements opérés } \\
\text { et nouvelles actions }\end{array}$ \\
\hline $\begin{array}{l}\text { Organiser mensuellement } \\
\text { les réunions des groupes } \\
\text { de travail } \\
\text { et trimestriellement } \\
\text { les réunions } \\
\text { des commissions } \\
\text { (NK; KOr) }\end{array}$ & $\begin{array}{l}\text { Une équipe plus cohésive } \\
\text { et plus performante } \\
\text { en appui aux districts } \\
\text { de santé et dans ses } \\
\text { interactions avec } \\
\text { les partenaires. } \\
\text { Plus d'alignement ou } \\
\text { d'adhésion des partenaires } \\
\text { aux priorités sanitaires } \\
\text { provinciales et } \\
\text { d'harmonisation des } \\
\text { stratégies d'action. }\end{array}$ & $\begin{array}{l}\text { Réunions régulières } \\
\text { et documentées } \\
\text { des groupes de travail. } \\
\text { Réunions régulières } \\
\text { et documentées } \\
\text { des commissions. }\end{array}$ & $\begin{array}{l}\text { Plus de cohésion au sein } \\
\text { de l'équipe et de crédibilité } \\
\text { vis-à-vis des districts } \\
\text { de santé; mais certaines } \\
\text { performances limitées. } \\
\text { Plus grande harmonisation } \\
\text { des stratégies d'action } \\
\text { et d'alignement } \\
\text { des partenaires. } \\
\text { Peu d'alignement des } \\
\text { partenaires d'urgence. }\end{array}$ & $\begin{array}{l}\text { Renforcer } \\
\text { la communication entre } \\
\text { les groupes de travail } \\
\text { et l'ensemble de l'équipe } \\
\text { provinciale. } \\
\text { Renforcer } \\
\text { la contractualisation avec } \\
\text { les partenaires d'urgence. }\end{array}$ \\
\hline $\begin{array}{l}\text { Organiser les supervisions } \\
\text { régulières et intégrées } \\
\text { des districts de santé (NK) }\end{array}$ & $\begin{array}{l}\text { Efficacité et efficience } \\
\text { dans l'accompagnement } \\
\text { des personnels } \\
\text { des districts de santé. }\end{array}$ & $\begin{array}{l}\text { Supervisions conjointes } \\
\text { avec des superviseurs } \\
\text { polyvalents et spécialisés. }\end{array}$ & $\begin{array}{l}\text { Efficience et crédibilité } \\
\text { du NISS vis-à-vis des cadres } \\
\text { des districts de santé. } \\
\text { Efficacité modérée pour } \\
\text { quelques programmes } \\
\text { spécialisés. }\end{array}$ & $\begin{array}{l}\text { Formation des superviseurs } \\
\text { par rapport aux } \\
\text { programmes spécialisés. } \\
\text { Structuration } \\
\text { de la préparation } \\
\text { des supervisions. }\end{array}$ \\
\hline $\begin{array}{l}\text { Charger la cellule de } \\
\text { gestion du NISS de la } \\
\text { gestion des fonds destinés } \\
\text { aux programmes verticaux } \\
\text { (NK) }\end{array}$ & $\begin{array}{l}\text { Plus de lisibilité } \\
\text { sur les financements } \\
\text { des activités. } \\
\text { Meilleure coordination } \\
\text { des activités. }\end{array}$ & $\begin{array}{l}\text { Gestion effective d'une } \\
\text { partie de fonds destinés } \\
\text { aux programmes } \\
\text { spécialisés. }\end{array}$ & $\begin{array}{l}\text { Meilleure lisibilité } \\
\text { sur les financements. } \\
\text { Capacités de } \\
\text { préfinancement des } \\
\text { activités améliorées. }\end{array}$ & $\begin{array}{l}\text { Systématiser la tenue } \\
\text { des réunions de monitoring } \\
\text { des financements. }\end{array}$ \\
\hline
\end{tabular}

sanitaires et la carte sanitaire font partie de ce dispositif pour la province du Nord Kivu. Dans la province du Kasaï oriental, un dispositif similaire à celui décrit pour le NISS Nord Kivu a été observé, avec quelques différences du point de vue de la dénomination des groupes de travail et de la fréquence des réunions, plus réduite.

\section{Le nouveau mode d'organisation des structures intermédiaires de santé en RDC}

Ce nouveau mode d'organisation est basé sur quatre métiers (figure 2) :

(i) Appui aux districts de santé ;

(ii) Gestion des ressources ;

(iii) Information sanitaire, recherche et communication et ;

(iv) Inspection-contrôle.

Le métier d'«Appui aux districts de santé » comprend (i) l'appui socio-technique aux districts de santé et (ii) l'intégration des programmes spécialisés. Le métier «Gestion des ressources » s'occupe de la gestion financière et de la contractualisation, de la gestion des ressources humaines, du charroi automobile et du matériel, de l'approvisionnement et de la logistique. Le métier
«Information sanitaire, communication et recherche » organise le système d'information sanitaire, structure la communication avec la population et les parties prenantes au développement sanitaire au niveau de la province. En outre, ce métier coordonne les processus de recherche. Le métier «Inspection - contrôle » s'intéresse à un large éventail de cibles dont le respect des normes et directives sanitaires provinciales et nationales (laboratoires, établissements de soins, établissements de distribution et vente de médicaments, écoles de formation des personnels de santé, établissements classés pour l'hygiène publique...). Les vérifications sont réalisées soit en routine, soit à la suite d'incidents, et donnent le plus souvent lieu à des mesures de régulation. La finalité est avant tout la protection de la santé des populations.

Ce mode d'organisation basé sur les quatre métiers est inspiré des dispositifs réels opérants, l'optique étant d'institutionnaliser une dynamique déjà existante et considérée comme efficace en la renforçant. Du point de vue fonctionnel, les quatre pôles de métiers sont articulés sur (i) cinq groupes de travail, (ii) une équipe cadre provinciale et (iii) un conseil de direction. Pour le NISS du Nord Kivu, les cinq groupes de travail sont: (i) la planification, (ii) l'information sanitaire, (iii) la surveillance épidémiologique et la gestion des urgences, (iv) l'encadrement/supervision, (v) l'approvisionnement et logistique (figure 2). 


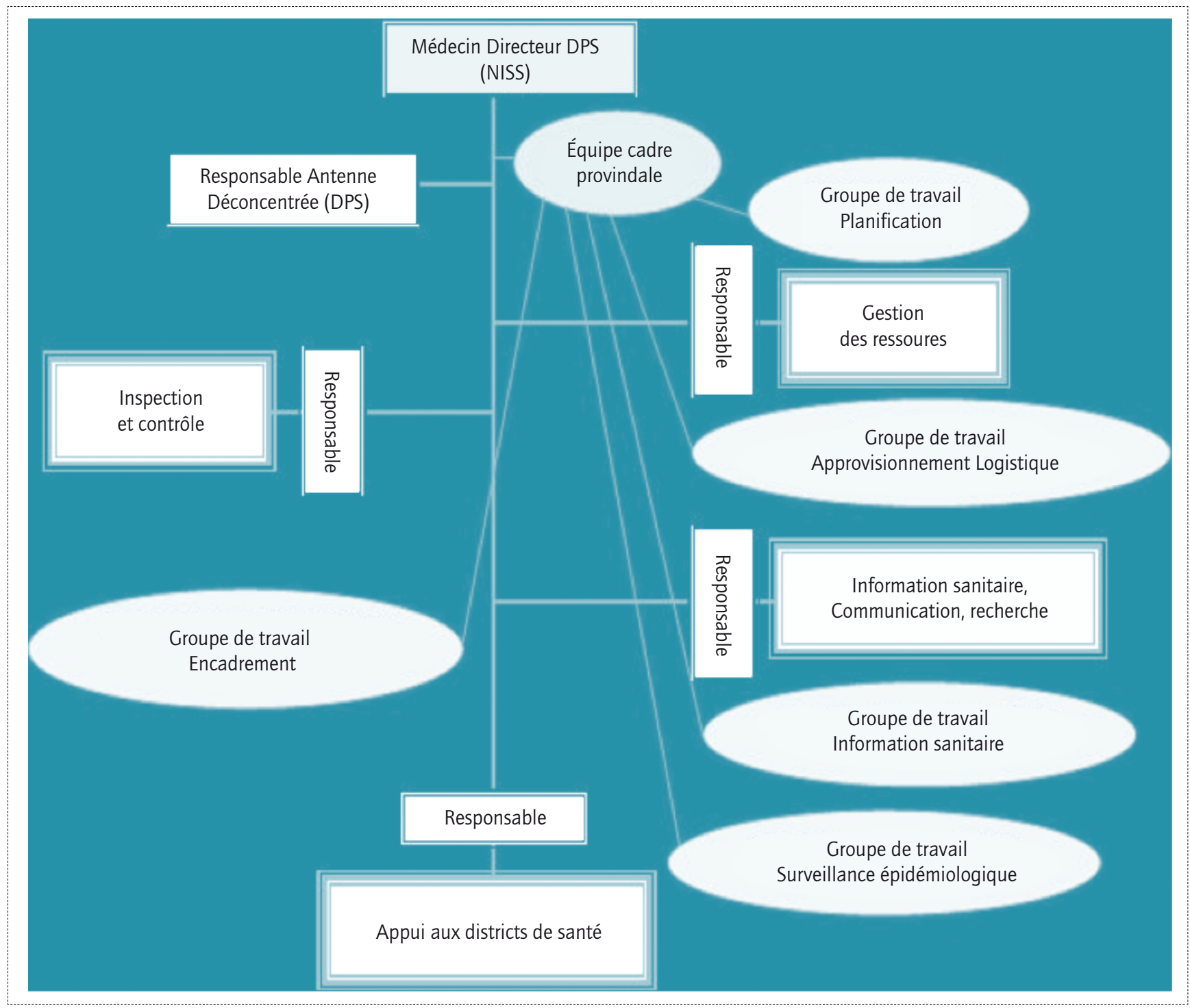

Figure 2 : Schéma synthétique du nouvel organigramme du NISS basé sur quatre métiers

Les groupes de travail sont des instances d'échanges, de résolution en interne de certaines questions problématiques et de réflexion sur certains projets. Les dénominations et le type de questions à traiter sont susceptibles de changer dans le temps et selon les provinces, en fonction des contextes et des problématiques auxquelles le NISS est confronté. Les groupes de travail sont connectés à des commissions, qui sont en fait des instances de concertation et d'échanges entre les cadres du NISS et les autres acteurs du système sanitaire. Les groupes de travail et les commissions sont coordonnés par les responsables des métiers.

Le conseil de direction est coordonné par le Médecin Directeur du NISS. Il est constitué de cadres responsables des métiers, qui sont en même temps responsables des groupes de travail, et du responsable d'une unité délocalisée ou antenne du NISS. Ce conseil a pour rôle d'inscrire une direction à la dynamique et de travailler à sa cohérence, son efficacité et à son efficience.

L'équipe cadre provinciale comprend les membres du conseil de direction ainsi que les autres cadres du NISS. Ses réunions constituent un espace d'échange d'informations et d'explicitation pour un partage plus large des options levées par le conseil de direction et les productions des groupes de travail.

À titre d'exemple, au Nord Kivu, la nouvelle structure a permis d'améliorer la régularité des supervisions du personnel de district, l'approvisionnement en médicaments essentiels et la coordination des interventions. 
Ces conditions, bien que non suffisantes, sont indispensables à la prestation des services de qualité au niveau des districts de santé. Entre 2008 et 2010, la couverture curative est passée de 0,50 à $0,67 \mathrm{NC} / \mathrm{hab}$, la couverture obstétricale de 79,2\% à 91,3\%. Les consultations prénatales ont été maintenues à plus 90,0\% et les couvertures vaccinales VAR et DTC3 à plus de $70 \%$.

Les résultats présentés au niveau national, en termes de métiers du NISS, ont été intégrés dans le nouveau plan de développement sanitaire 2011-2015 de la RDC et sont en voie de généralisation dans l'entièreté du pays.

\section{Discussion}

Cette recherche-action a permis de mettre au point un nouveau mode d'organisation du NISS. Nous proposons de discuter en quoi il est compatible avec le pilotage et la régulation d'un système sanitaire complexe, et d'examiner son potentiel de soutien à la revitalisation des districts de santé ainsi que les défis de sa mise en œuvre en RDC.

\section{Potentiel de pilotage et de régulation d'un système sanitaire complexe}

L'approche systémique de renforcement des systèmes de santé met l'accent sur les interrelations entre les éléments constitutifs et les synergies nécessaires pour améliorer ses performances [16]. Les instances d'échanges, d'une part en interne entre les personnels de différents métiers au niveau du NISS (conseil de direction, équipe cadre, groupes de travail) et d'autre part en externe, entre le NISS et les autres acteurs (commissions, comité de pilotage) offrent plusieurs opportunités de synergies : (i) l'expression des besoins des cadres du NISS et d'autres acteurs, (ii) la participation à la prise de décision, (iii) la participation à la prise d'initiatives, (iv) le captage et la production d'informations, et enfin (v) un apprentissage individuel et collectif. Ces éléments sont essentiels pour un pilotage sanitaire efficace [3] et propices à une gouvernance qui s'adapte au contexte.

\section{Potentiel de soutien à la revitalisation du district de santé en RDC}

Le potentiel de soutien au district de santé est à rechercher au niveau (i) de l'adéquation entre les métiers et les besoins des districts de santé, (ii) des compétences du personnel du NISS et (iii) de la gestion des interfaces avec les autres acteurs.

\section{(i) Adéquation des métiers par rapport aux besoins de revitalisation des districts de santé}

\section{- Le métier d'Appui aux districts de santé}

Les deux volets de ce métier ((i) l'appui socio-technique aux districts de santé et (ii) l'intégration des programmes spécialisés) rejoignent globalement les responsabilités d'encadrement du personnel, les supervisions et les formations du personnel décrits par Vaughan [17]. Ils rejoignent également l'appui technique aux districts de santé décrit dans le document des normes du Ministère de la Santé de la RDC [18] qui a été repris dans sa stratégie de réforme [8]. Le deuxième volet de ce métier répond à la nécessité de soutenir l'intégration des activités des programmes spécialisés pour améliorer la performance desdits programmes [19]. La dimension « socio-technique » suppose la prise en compte de l'interdépendance entre la dimension sociale et la dimension technique [20]. Des aspects humains comme l'amélioration de l'environnement et des conditions de travail du personnel, la qualité des relations humaines, l'estime de soi, doivent être renforcés conjointement aux capacités techniques pour espérer une amélioration durable des performances du personnel [21]. Des études dans d'autres contextes ont montré l'importance des supervisions dans l'amélioration de la motivation et des performances du personnel de santé [22,23]. Dans ces conditions, ce métier contribue à renforcer les piliers "leadership et gouvernance ", "ressources humaines" et "prestations des services » $[16,24]$ au niveau des districts de santé.

\section{- Le métier "Gestion des ressources"}

Ce métier est essentiel à l'optimisation de la gestion interne du NISS et au renforcement de sa crédibilité vis-àvis des autres acteurs. Comme le souligne Vaughan [17], ce volet est primordial pour le district de santé pour opérer l'acquisition des équipements et des médicaments sur le marché international et par l'économie d'échelle qu'il permet en regroupant les commandes [25]. Ce métier renvoie aux piliers "système de financement», "vaccins, technologies et produits médicaux» et "ressources humaines » $[16,24]$ au niveau du district de santé.

\section{- Le métier "Information sanitaire, communication et recherche"}

Ugwuegbu a reproché aux organisations publiques africaines une gestion non établie sur des bases factuelles et 
l'information que l'on en extrait [26]. En 2003, l'OMS a stigmatisé la sous-utilisation de l'information dans la gestion des systèmes de santé locaux, tout en soulignant son importance [27]. Ce métier met en exergue l'importance de l'information dans la gestion des services de santé et rejoint les préoccupations formulées par la RDC dans sa réforme du système sanitaire, à savoir le renforcement du système d'information sanitaire et de la recherche $[8,9]$. Traitant de la gouvernance des systèmes sanitaires, Siddiqi et al. ont souligné le rôle primordial de l'information dans le pilotage du secteur sanitaire [2]. Tous ces éléments indiquent le rôle indispensable de ce métier comme condition à l'exercice des autres métiers du NISS et dans le renforcement des six piliers au niveau du district de santé.

\section{- Le métier "Inspection - contrôle"}

Les éléments de ce métier ont été clairement invoqués par Vaughan en 1991 [17] ; ils contribuent à l'exercice des fonctions régulatrices du système sanitaire dans la province par le Niveau intermédiaire.

\section{(ii) Compétences du personnel}

La performance globale du NISS tient aussi aux compétences des personnes qui en font partie. Ces compétences supposent des savoirs, des aptitudes et des attitudes qui favorisent l'efficacité dans les responsabilités individuelles, les interactions entre personnels au sein d'un métier et les synergies entre les différents métiers. Le système étant dynamique, les attentes des acteurs sont susceptibles de varier. Ainsi les compétences doivent sans cesse être renouvelées pour s'adapter et répondre au mieux aux besoins et aux demandes des acteurs; d'où la nécessité d'un apprentissage continu. Cette approche rejoint la description de Laville [28] au sujet du nouveau modèle professionnel : les acteurs, face à l'incertitude des contextes et de la demande, sont amenés à réaliser des tâches qui requièrent des savoirs et des savoir-faire non stabilisés. Cet apprentissage continu requiert un environnement propice, comme celui décrit par Bériot [29], un environnement favorisant l'expression et la proposition par le personnel, des valeurs, des objectifs individuels et collectifs, des modifications des processus, des innovations et des savoir-faire. Les groupes de travail, les réunions de l'équipe provinciale et le conseil de direction contribuent à cet environnement décrit par Bériot; ils apportent la composante adhocratique à ce nouveau mode d'organisation, configuré en adhocratie professionnelle (figure 3).

\section{(iii) Gestion des interfaces avec les autres acteurs}

Le nombre important d'acteurs et de parties prenantes au développement du secteur de la santé au niveau d'une province impose une gestion professionnelle des interfaces. La première interface concerne les équipes de gestion des districts de santé. Le métier d'appui aux districts de santé est dédié à cette première catégorie d'acteurs. Les autres métiers interviennent également, bien que dans une moindre mesure, dans la gestion de cette interface. La deuxième interface concerne les partenaires techniques et financiers du secteur santé ainsi que les acteurs de la société civile. Cet interface implique surtout les métiers i) de gestion et ii) d'information, communication et recherche. La communication des organisations de santé avec le public est souvent sous-estimée. Structurée, elle contribue à renforcer la participation des communautés à l'action sanitaire. L'expérience de certaines régions canadiennes qui ont systématisé l'information de la population [30] est illustrative de la qualité de la participation à l'action sanitaire induite par une communication de qualité. La troisième interface concerne les autorités centrales et provinciales. Le conseil de direction, le métier de gestion et le métier de contrôle et inspection sont les plus impliqués dans la gestion de cette interface. Au-delà de la contribution des métiers à la gestion de ces trois interfaces spécifiques, le comité provincial de pilotage de l'action sanitaire, ses commissions, les revues (annuelle, semestrielle) des activités des soins de santé primaires et les conseils d'administration des districts de santé, constituent des instances d'interface plus générales entre l'équipe du Niveau intermédiaire et les autres acteurs.

\section{Défis pour la mise en œuvre du nouveau mode d'organisation du NISS}

Ce nouveau mode d'organisation relève plus d'une hypothèse de travail que du prescriptif pour d'autres provinces de la RDC. Son implémentation dans d'autres provinces requiert une adaptation aux contextes et aux problématiques qui leur sont propres. En outre, elle impose un minimum de financements, de progressivité dans sa mise en œuvre, de flexibilité et de changement dans les logiques des parties prenantes. Elle est aussi liée à la poursuite d'autres réformes amorcées dans le secteur de la santé en RDC (réforme du niveau central, du financement de la santé) et des secteurs connexes à la santé. 


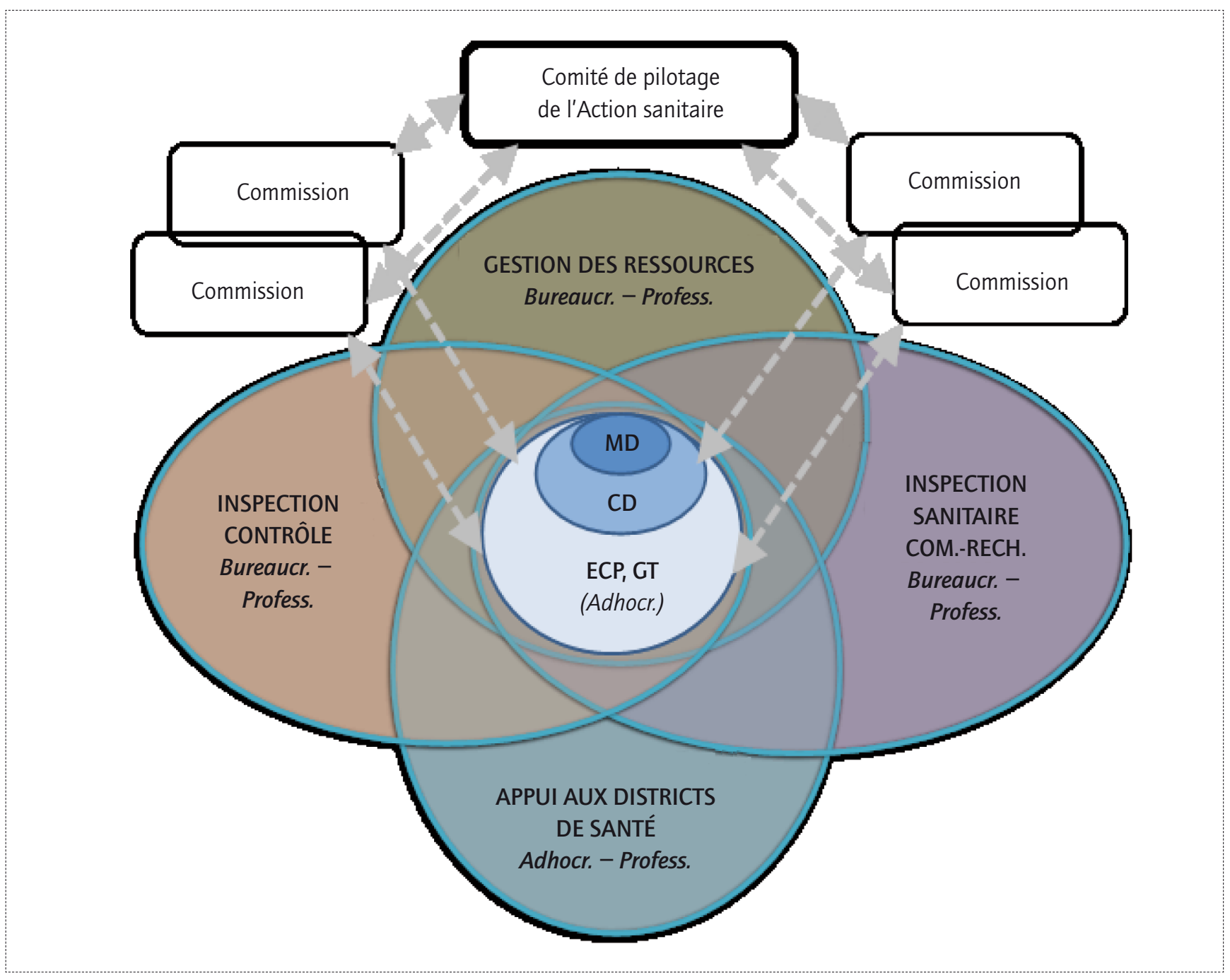

Figure 3 : Modélisation du nouveau mode d'organisation du NISS en RDC

\section{Conclusions}

La recherche-action participative constitue selon nous un outil essentiel du processus de transformation des systèmes de santé dans un contexte complexe et fragile. Cette étude aura montré qu'il est possible d'impliquer les acteurs de terrain dans un processus qui intègre en même temps la résolution des problèmes concrets, la production des savoirs contextualisés et la transformation organisationnelle. La transformation des structures intermédiaires de santé suggérée et testée dans le cadre de cette étude est une adhocratie professionnelle. Cette forme organisationnelle est flexible et propice à l'exercice des fonctions de gouvernance adaptative et de soutien sociotechnique et logistique aux districts de santé. Elle est également plus propice à intégrer les principes de subsidiarité et d'apprentissage organisationnel. Ces deux principes sont indispensables pour une organisation de pilotage et de régulation d'un niveau du système sanitaire dans un environnement complexe. Ils permettent effectivement d'envisager un changement organisationnel profond au bénéfice des systèmes de santé de district, y compris dans les pays dits "fragiles», en situation de crise chronique. 


\section{Références}

1. Organisation Mondiale de la Santé (OMS). Rapport sur la santé dans le monde. Soins de santé primaires : maintenant plus que jamais. Genève : OMS ; 2008, 125 p.

2. Siddiqi S, Masud TI, Nishtar S, Peters DH, Sabri B, Bile KM et al. Framework for assessing governance of the health system in developing countries: gateway to good governance. Health Policy. 2009;90:13-25.

3. Veillard JHM, Brown AD, Barıs E Permanand G, Klazinga NS. Health system stewardship of national health ministries in the WHO European region: concepts, functions and assessment framework. Health Policy. 2011;103:191-9.

4. Unger JP, Macq J, Bredo F, Boelaert M. Through Mintzberg glasses: a fresh look at the organization of ministries of health. Bull World Heath Organ. 2000;78:1005-14.

5. Blaise P, Kegels G. A realistic approach to the evaluation of the quality management movement in health care systems: a comparison between European and African contexts based on Mintzberg's organizational models. Int J Health Plann Manage. 2004;19:337-64.

6. Unger JP, De Paepe P, Gilbert P, Soors W, Green A. Integrated care: a fresh perspective for international health policies in low and middleincome countries. Int J Integr Care. 2006;6:e15.

7. Organisation Mondiale de la santé(OMS). Rapport sur la santé dans le monde, 2000 : pour un système de santé plus performant. Genève: OMS ; 2000. 248 p. [Visité le 16/08/2014]. En ligne: http://www.who.int/whr/2000/en/whr00_fr.pdf?ua=1.

8. République Démocratique du Congo, Ministère de la santé publique, Secrétariat général. Stratégie de renforcement du système de santé

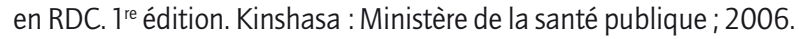
$49 \mathrm{p}$.

9. République Démocratique du Congo, Ministère de la santé publique, Secrétariat général. Stratégie de renforcement du système de santé en RDC. 2e édition. Kinshasa : Ministère de la santé publique ; 2010. $67 \mathrm{p}$.

10. Parent $F$, Ndiaye $M$, Coppieters $Y$, Deme $S$, Sarr O, Lejeune $C$ et al. Utilisation originale de l'approche par compétences en supervision formative en Afrique subsaharienne. Pédagogie Médicale. 2007:8:156-76.

11. Nonaka I, Takeuchi H. La connaissance créatrice, la dynamique de I'entreprise apprenante. Bruxelles: De Boeck Université ; 1997. $303 \mathrm{p}$.

12. Grodos $D$, Mercenier $P$. Recherche sur les systèmes de santé : mieux comprendre la méthodologie pour mieux agir. Anvers (BEL) : ITG Press; 2000. 112 p. (Studies in health services and organization and policy; $\left.n^{\circ} 14\right)$. [Visité le 23/08/2014]. En ligne : http://www.itg. be/itg/generalsite/infservices/downloads/shsop14.pdf.
13. Koenig G. L'apprentissage organisationnel : repérage des lieux. Revue française de gestion. 2006;160:293-306.

14. Bonami M. Une entreprise peut-elle apprendre ? Apprentissage des personnes et/ ou apprentissage organisationnel. Contact. 2008; 72: 4 p. [Visité le 23/08/2014]. En ligne: http://www.cfip.be/ data/1200317127Contact_janvier_2008.pdf.

15. Le Boterf G. Repenser la compétence. Paris : Éditions d'Organisation; 2008. 140 p.

16. De Savigny D, Adam T. Pour une approche systémique de renforcement des systèmes de santé : Alliance pour la recherche sur les politiques et les systèmes de santé. Genève : OMS ; 2009. 113 p.

17. Vaughan JP. Leçons de l'expérience. In: Mills A, Vaughan JP, Smith LD, Tabibzadeh I ; éditeurs. Décentralisation des systèmes de santé. concepts, problèmes et expériences de quelques pays. Genève : OMS ; 1991. 174 p.

18. République Démocratique du Congo, Ministère de la Santé. Recueil des normes de la Zone de santé. Kinshasa: Ministère de la Santé ; 2001. 80 p.

19. Unger JP, De Paepe P, Gilbert P, Soors W, Green A. Disintegrated care: the Achilles heel of international health policies in low and middleincome countries. Int J Integr Care. 2006;6:e14.

20. Rojot, J. Théorie des organisations. Paris : Eska ; 2003. 478 p.

21. Organisation Mondiale de la santé (OMS). Travailler ensemble pour la santé. Rapport sur la santé dans le monde. Genève : OMS ; 2006. $215 \mathrm{p}$.

22. Bosch-Capblanch $X$, Garner P. Primary health care supervision in developing countries. Trop Med Int Health. 2008;13(3):369-83.

23. Rowe AK, de Savigny D, Lanata CF, Victora CG. How can we achieve and maintain high-quality performance of health workers in lowresource setting? Lancet. 2004;366:1026-35.

24. World Health Organization (WHO). Everybody's Business: strengthening health systems to improve health outcomes: WHO framework for action. Genève: WHO; 2007. 56 p. [Visité le 23/08/2014]. En ligne : http://www.who.int/healthsystems/strategy/everybodysbusiness.pdf.

25. Bossert TJ, Bowser DM, Amenyah JK. Is decentralization good for logistics systems? Evidence on essential medicine logistics in Ghana and Guatemala. Health Policy Plan. 2008;22(2):73-82.

26. Ugwuegbu DCE. The psychology of management in African organizations. Westport (CT): Quorum Books; 2001. 286 p.

27. Organisation Mondiale de la Santé (OMS). Rapport sur la santé dans le monde 2003. Façonner l'avenir. Genève : OMS ; 2003. 193 p.

28. Laville JL. Sociologie des services: entre marché et solidarité. Ramonville Saint-Agne: Erès; 2005. 180 p. (Sociétés en changement).

29. Bériot D. Manager par l'approche systémique : s'approprier le nouveau savoir-faire pour agir dans la complexité. Paris : Éditions d'Organisation; 2006.340 p.

30. Denis JL. Gouvernance et gestion du changement dans le système de santé au Canada. Montréal : Commission sur l'avenir des soins de santé au Canada ; 2002. 39 p. (Étude ; n³6). 